\title{
Rademacher Functions and Their Applications to 3GPP Mobile Communication Systems
}

\author{
Young Joon Song \\ Department of Electronic Engineering \\ Kumoh National Institute of Technology, \\ 1 Yangho-dong, Gumi, Gyungbuk, 730-701, Korea \\ yjsong@kumoh.ac.kr
}

\begin{abstract}
In this paper we investigate the applications of Rademacher functions in 3GPP mobile communication systems which are currently most widely being deployed in the world. Although the equations are simple enough to be expressed as signs of sine functions, it is very hard to find the limit of the usefulness in modern communication systems such as $3 G P P$ and $3 G P P 2$ society. In this paper we narrow the scope of the applications to 3GPP mobile communication systems for the better understanding, where the functions are used for OVSF code, TFCI and CQI codings.
\end{abstract}

Keywords: Rademacher functions, OVSF code, TFCI coding, CQI coding

\section{Introduction}

In 3GPP W-CDMA (Wideband Code Division Multiple Access) system, DPCCH (Dedicated Physical Control Channel) uses the TFCI (Transport Format Combinaion Indicator) coding to tramsmit TFI (Transport Format Indicator) information with error protection capability [1]-[3]. The transport channel is accompanied by TFI and the physical layer combines the TFI information from different transport channels to TFCI which is transmitted on DPCCH. For the downlink if one of DCH (Dedicated Channel) is associated with a DSCH (Downlink Shared Channel), $(16,5)$ TFCI coding is used in split mode [3]-[4]. Considering the simplity of encoding and decoding of the TFCI coding with the optimum BER performance, 3GPP mobile communication systems decided to adopt the 1st order and 2nd order RM codes as the coding scheme of TFCI. The basis vectors of the RM codes are specially desinged to have maximized minimum Hamming distance. The basis sequences of the TFCI coding is based on the Rademacher functions.

The uplink feedback information corresponding to the HS-DSCH such as HARQ feedback and CQI (Channel Quality Indicator) is carried on the HS-DPCCH (High Speed Dedicated Physical Control Channel). HARQ feedback is ACK/NACK for the received packet of HS-DSCH and the CQI informs the base station scheduler of the date rate that the terminal is able to receive at a given time. The HS-DSCH is transport channel which is mapped on HS-PDSCH (High Speed Physical Downlink Shared Channel) and carries the user data with HSDPA (High Speed Downlink Packet Access) [5]. The CQI coding of HS-DPCCH (High Speed Dedicated Physical Control Channel) are effectively equivalent to those of $(16,5)$ TFCI (Transport Format Combinaion Indicator) coding in W-CDMA system.

The OVSF (Orthogonal Variable Spreading Factor) codes of W-CDMA system which are used for channel allocation of traffic and control channels can be generated from Rademacher functions. It preserves orthogonality between these control and data channels and used to seperate data and control channels from the same UE (User Equipment) [4-5]. Thus, using the OVSF codes we can adjust the spreading facotr to be variable and maintain the orthogonality between different spreading codes of different lengths [6-7]. 
Investing the Rademacher function, we can easily find the similarity and commonality between OVSF and TFCI codings of W-CDMA system and CQI codings of HSDPA system in 3GPP FDD mode and TDD mode specifications.

\section{Rademacher Functions}

The Rademacher functions which have many applications in 3GPP mobile communication shystem are expressed as [8]:

$$
\mathrm{R}_{n}(t)=\operatorname{sgn}\left(\sin \left(2^{n} \pi t\right)\right)
$$

, where $t \in(0,1), n=1,2, \ldots, \log _{2} N=K$, and

$$
\operatorname{sgn}(x)=\left\{\begin{array}{c}
+1, x>0 \\
0, x=0 \\
-1, x<0
\end{array}\right.
$$

and $\mathrm{R}_{0}(t)=+1$. Table 1 and 2 show the Rademacher functions expressed as zeros or ones after mapping $0 \leftrightarrow+1,1 \leftrightarrow-1$ with $N=2^{K}$, where $K=1,2,3,4,5,6$.

Table 1. Rademacher Functions with $\mathrm{K}=1,2,3,4,5$

\begin{tabular}{|c|c|c|}
\hline$N$ & $K$ & $\mathrm{R}_{n}(t)$ \\
\hline 2 & 1 & $\mathrm{R}_{1}(t)=(01)$ \\
\hline 4 & 2 & $\mathrm{R}_{1}(t)=(0011)$ \\
$\mathrm{R}_{2}(t)=(0101)$
\end{tabular}


Table 2. Rademacher functions with $\mathrm{K}=6$

\begin{tabular}{|c|}
\hline$N=64, K=6$ \\
\hline $\mathrm{R}_{1}(t)=(0000000000000000000000000000000011111111111111111111111111111111)$ \\
$\mathrm{R}_{2}(t)=(0000000000000000111111111111111100000000000000001111111111111111)$ \\
$\mathrm{R}_{3}(t)=(000000001111111100000000111111110000000011111111000000001111111)$ \\
$\mathrm{R}_{4}(t)=(0000111100001111000011110000111100001111000011110000111100001111)$ \\
$\mathrm{R}_{5}(t)=(0011001100110011001100110011001100110011001100110011001100110011)$ \\
$\mathrm{R}_{6}(t)=(0101010101010101010101010101010101010101010101010101010101010101)$
\end{tabular}

\section{OVSF and TFCI Codes using Rademacher Functions}

\subsection{OVSF Coding}

Figure 1 shows the code tree to generate OVSF codes in W-CDMA system [6-7]. Figure 2 shows the OVFS code generator using Rademacher functions as the basis sequences. This implies that the OVSF codes are linear combination of Rademacher functions. The OVSF code $C_{\mathrm{i}}^{N}$ is generated by setting $K=\log _{2} N$ binary bits such as $i=\left(d_{0}, d_{1}, \ldots, d_{K-1}\right)_{2}$ which is the unsigned integer such that $i=\sum_{z=0}^{K-1} d_{z} 2^{z}$.

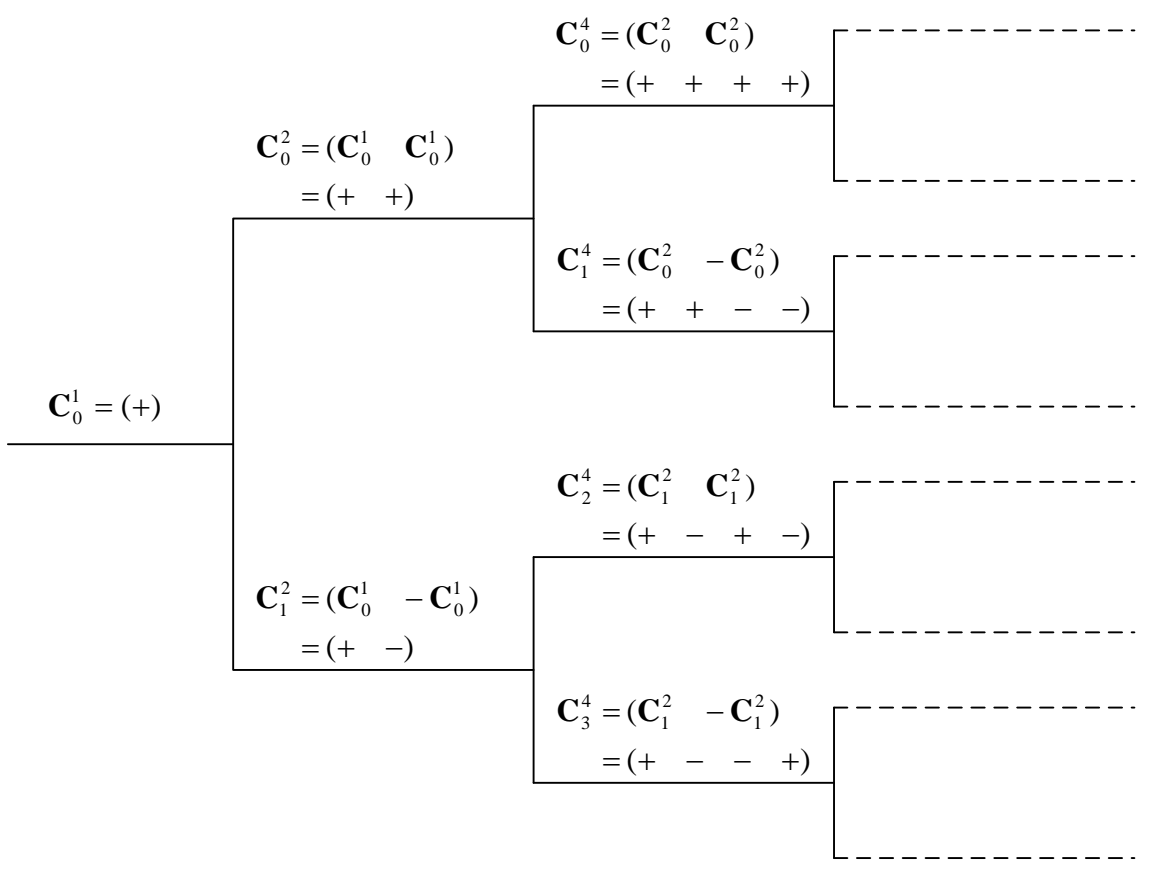

Figure 1. Code Tree to Generate OVSF Codes 


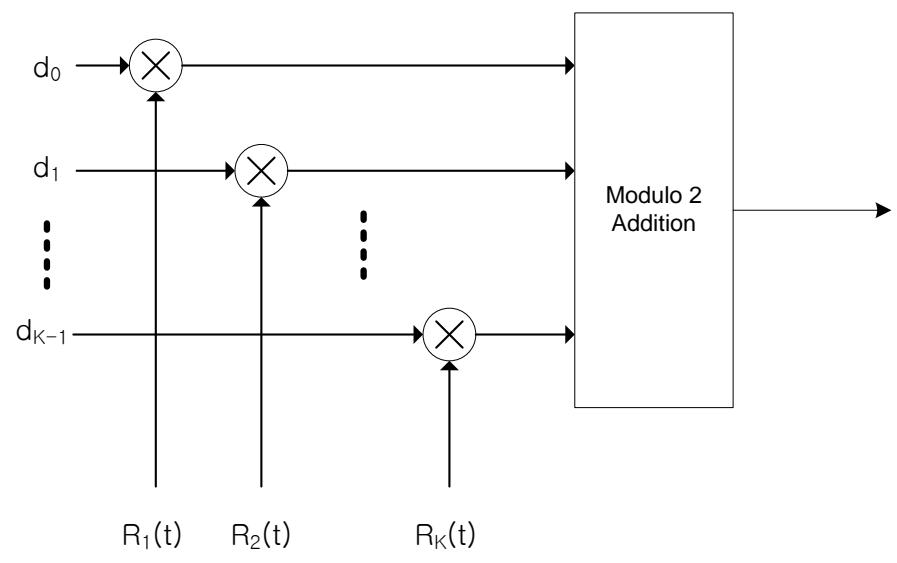

Figure 2. Generation of OVSF Codes using Rademacher Functions

\subsection{TFCI Coding}

\subsection{1 (32, 10) TFCI Coding}

In 3GPP FDD mode also called W-CDMA system, DPCCH uses the TFCI coding to tramsmit TFI information. Figure 3 shows $(32,10)$ TFCI encoder to encode TFCI bits expressed by $a_{0}, a_{1}, \ldots, a_{n-1}, 1 \leq n \leq 10$, which are linearly combined with the basis sequences of Table 3 [1]-[3]. The number of TFCI bits is determined by upper layer. If the number of input TFCI bits is 1 to 4, the last symbol of TFCI code word of length 16 is always all " 0 ". Thus in this case, puncturing the last symbol does not decrease the minimum Hamming distance. When the number of input TFCI bits is 5, puncturing the last 2 symbols decreases the minimum Hamming distance by 1 [9]-[12]. Figure4 illustrates how the basis sequences of $(32,10)$ TFCI code are made from the Rademacher functions. First the 0 and $16^{\text {th }}$ bits of Rademacher functions are moved to the last two bits position. Second the Rademacher functions $\left\{\mathrm{R}_{1}(\mathrm{t}), \mathrm{R}_{2}(\mathrm{t}), \mathrm{R}_{3}(\mathrm{t}), \mathrm{R}_{4}(\mathrm{t}), \mathrm{R}_{5}(\mathrm{t})\right\}$ are mapped to $\left\{M_{4}(t), M_{3}(t), M_{2}(t), M_{1}(t), M_{0}(t)\right\}$ using barrel shifting [9]-[10]. The basis sequence $\mathrm{M}_{5}(\mathrm{t})$ is all " 1 " pattern for bi-orthogonal coding. After linear combination of $\left\{\mathrm{M}_{0}(\mathrm{t}), \mathrm{M}_{1}(\mathrm{t}), \mathrm{M}_{2}(\mathrm{t}), \mathrm{M}_{3}(\mathrm{t}), \mathrm{M}_{4}(\mathrm{t})\right\} \quad$, we obtain the basis sequences $\left\{M_{6}(t), M_{7}(t), M_{8}(t), M_{9}(t)\right\}$ for $2^{\text {nd }}$ order Reed Muller code. The $(32,10)$ TFCI coding is also used for 3GPP TDD mode which is called TD-CDMA system.

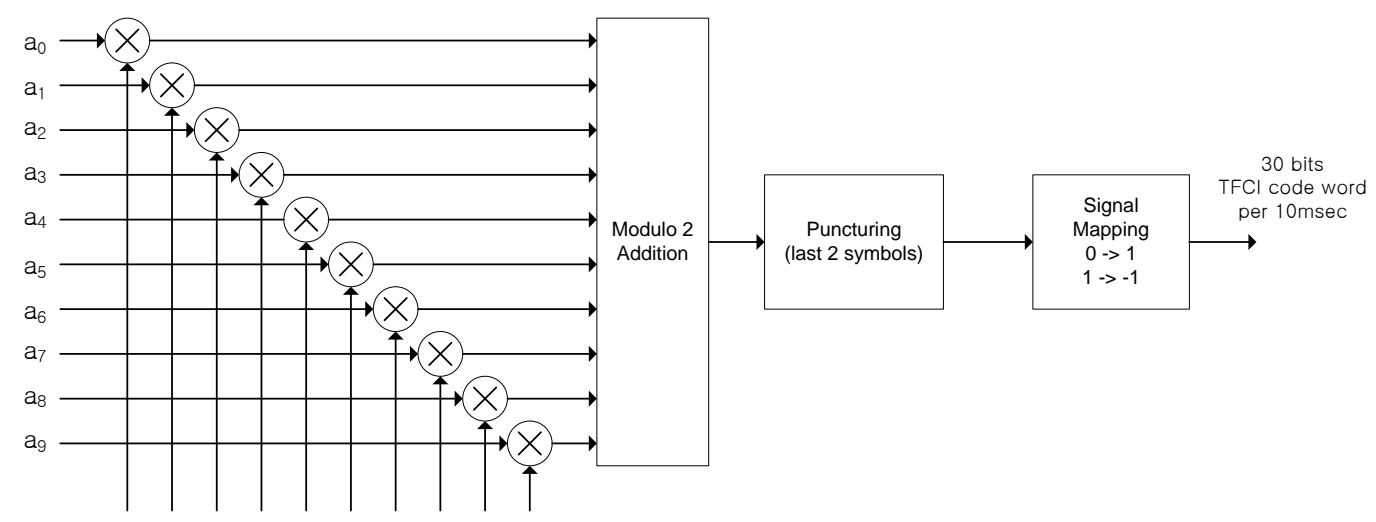

$\begin{array}{llllllllll}M_{0} & M_{1} & M_{2} & M_{3} & M_{4} & M_{5} & M_{6} & M_{7} & M_{8} & M_{9}\end{array}$

Figure 3. $(32,10)$ TFCI Encoder 


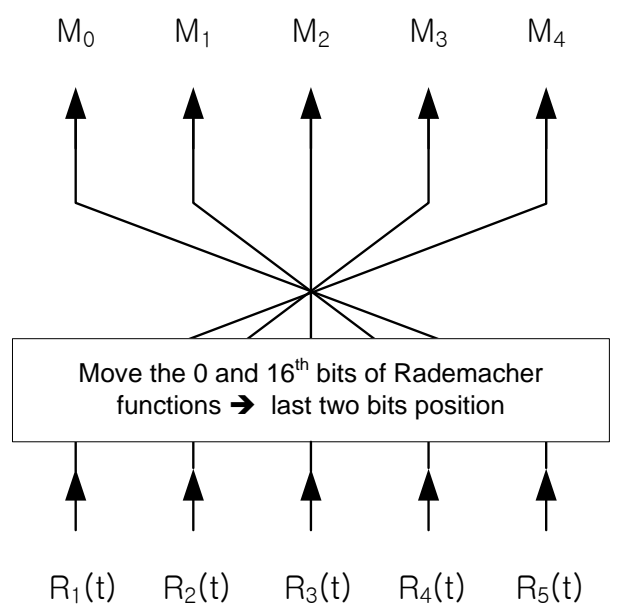

Figure 4. Barrel shift of Rademacher Functions for $(32,10)$ TFCI Code

Table 3. Basis Sequences of $(32,10)$ TFCl code

\begin{tabular}{|c|c|c|c|c|c|c|c|c|c|}
\hline $\mathrm{M}_{0}$ & $\mathrm{M}_{1}$ & $\mathrm{M}_{2}$ & $\mathrm{M}_{3}$ & $\mathrm{M}_{4}$ & $\mathrm{M}_{5}$ & $\mathrm{M}_{6}$ & $\mathrm{M}_{7}$ & $\mathrm{M}_{8}$ & $\mathrm{M}_{9}$ \\
\hline 1 & 0 & 0 & 0 & 0 & 1 & 0 & 0 & 0 & 0 \\
0 & 1 & 0 & 0 & 0 & 1 & 1 & 0 & 0 & 0 \\
1 & 1 & 0 & 0 & 0 & 1 & 0 & 0 & 0 & 1 \\
0 & 0 & 1 & 0 & 0 & 1 & 1 & 0 & 1 & 1 \\
1 & 0 & 1 & 0 & 0 & 1 & 0 & 0 & 0 & 1 \\
0 & 1 & 1 & 0 & 0 & 1 & 0 & 0 & 1 & 0 \\
1 & 1 & 1 & 0 & 0 & 1 & 0 & 1 & 0 & 0 \\
0 & 0 & 0 & 1 & 0 & 1 & 0 & 1 & 1 & 0 \\
1 & 0 & 0 & 1 & 0 & 1 & 1 & 1 & 1 & 0 \\
0 & 1 & 0 & 1 & 0 & 1 & 1 & 0 & 1 & 1 \\
1 & 1 & 0 & 1 & 0 & 1 & 0 & 0 & 1 & 1 \\
0 & 0 & 1 & 1 & 0 & 1 & 0 & 1 & 1 & 0 \\
1 & 0 & 1 & 1 & 0 & 1 & 0 & 1 & 0 & 1 \\
0 & 1 & 1 & 1 & 0 & 1 & 1 & 0 & 0 & 1 \\
1 & 1 & 1 & 1 & 0 & 1 & 1 & 1 & 1 & 1 \\
1 & 0 & 0 & 0 & 1 & 1 & 1 & 1 & 0 & 0 \\
0 & 1 & 0 & 0 & 1 & 1 & 1 & 1 & 0 & 1 \\
1 & 1 & 0 & 0 & 1 & 1 & 1 & 0 & 1 & 0 \\
0 & 0 & 1 & 0 & 1 & 1 & 0 & 1 & 1 & 1 \\
1 & 0 & 1 & 0 & 1 & 1 & 0 & 1 & 0 & 1 \\
0 & 1 & 1 & 0 & 1 & 1 & 0 & 0 & 1 & 1 \\
1 & 1 & 1 & 0 & 1 & 1 & 0 & 1 & 1 & 1 \\
0 & 0 & 0 & 1 & 1 & 1 & 0 & 1 & 0 & 0 \\
1 & 0 & 0 & 1 & 1 & 1 & 1 & 1 & 0 & 1 \\
0 & 1 & 0 & 1 & 1 & 1 & 1 & 0 & 1 & 0 \\
1 & 1 & 0 & 1 & 1 & 1 & 1 & 0 & 0 & 1 \\
0 & 0 & 1 & 1 & 1 & 1 & 0 & 0 & 1 & 0 \\
1 & 0 & 1 & 1 & 1 & 1 & 1 & 1 & 0 & 0 \\
0 & 1 & 1 & 1 & 1 & 1 & 1 & 1 & 1 & 0 \\
1 & 1 & 1 & 1 & 1 & 1 & 1 & 1 & 1 & 1 \\
0 & 0 & 0 & 0 & 0 & 1 & 0 & 0 & 0 & 0 \\
0 & 0 & 0 & 0 & 1 & 1 & 1 & 0 & 0 & 0 \\
\hline & & & & & & & & & \\
\hline
\end{tabular}

\subsection{2 (16,5) TFCI coding}

Figure 5 shows $(16,5)$ TFCI encoder to encode TFCI bits expressed by $a_{0}, a_{1}, \ldots, a_{n-1}$, $1 \leq n \leq 5$, which are linearly combined with the basis sequences of Table 4 . The number 
of TFCI bits is determined by upper layer. If the number of input TFCI bits is 1 to 4 , the last symbol of TFCI code word of length 16 is always all " 0 ". Thus in this case, puncturing the last symbol does not decrease the minimum Hamming distance [9]-[12]. Figure 6 illustrates how the basis sequences of $(16,5)$ TFCI code are constructed from the Rademacher functions with $K=4$ of Table 1 . First the 0th bits of Rademacher functions are moved to the last positions of the functions. Second the functions $\left\{\mathrm{R}_{1}(\mathrm{t}), \mathrm{R}_{2}(\mathrm{t}), \mathrm{R}_{3}(\mathrm{t}), \mathrm{R}_{4}(\mathrm{t})\right\}$ are mapped to sequences $\left\{\mathrm{M}_{3}(\mathrm{t}), \mathrm{M}_{2}(\mathrm{t}), \mathrm{M}_{1}(\mathrm{t}), \mathrm{M}_{0}(\mathrm{t})\right\}$, respectively [9]-[10]. The basis sequence $\mathrm{M}_{4}(\mathrm{t})$ is all " 1 " for bi-orthogonal coding.

When we encode the short TFCI in 3GPP TDD, if the number of TFCI bits is 1 or 2, then repetition will be used for TFCI encoding and if the unber of TFCI bits is in the range of 3 to 5, the TFCI bits are encoded using $(16,5)$ TFCI coding of Figure 5 with basis sequences of Table 4 [2].

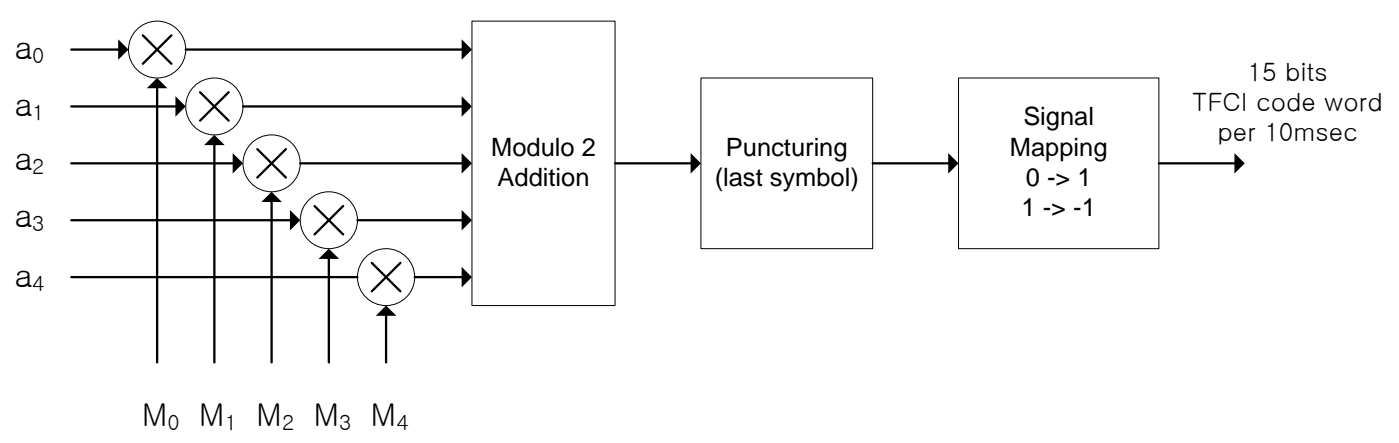

Figure 5. $(16,5)$ TFCl encoder

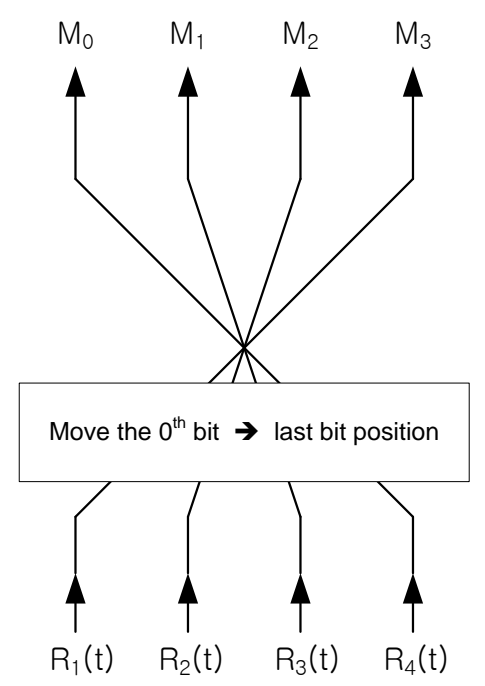

Figure 6. Generation of Basis Sequences for $(16,5)$ TFCI Code 
Table 4. Basis Sequences of $(16,5)$ TFCI Code

\begin{tabular}{|c|c|c|c|c|}
\hline $\mathrm{M}_{0}$ & $\mathrm{M}_{1}$ & $\mathrm{M}_{2}$ & $\mathrm{M}_{3}$ & $\mathrm{M}_{4}$ \\
\hline 1 & 0 & 0 & 0 & 1 \\
0 & 1 & 0 & 0 & 1 \\
1 & 1 & 0 & 0 & 1 \\
0 & 0 & 1 & 0 & 1 \\
1 & 0 & 1 & 0 & 1 \\
0 & 1 & 1 & 0 & 1 \\
1 & 1 & 1 & 0 & 1 \\
0 & 0 & 0 & 1 & 1 \\
1 & 0 & 0 & 1 & 1 \\
0 & 1 & 0 & 1 & 1 \\
1 & 1 & 0 & 1 & 1 \\
0 & 0 & 1 & 1 & 1 \\
1 & 0 & 1 & 1 & 1 \\
0 & 1 & 1 & 1 & 1 \\
1 & 1 & 1 & 1 & 1 \\
0 & 0 & 0 & 0 & 1 \\
\hline
\end{tabular}

\subsection{3 (48, 10) TFCI Coding}

TFCI encoding depends on the number of TFCI bits and the modulation in use [1-3]. When 2Mcps service is transmitted, 8PSK mdoulation is applied in 1.28Mcps TDD option. The $(48,10)$ TFCI coding is used when the nuber of TFCI bits is in the rage of 6 to 10.

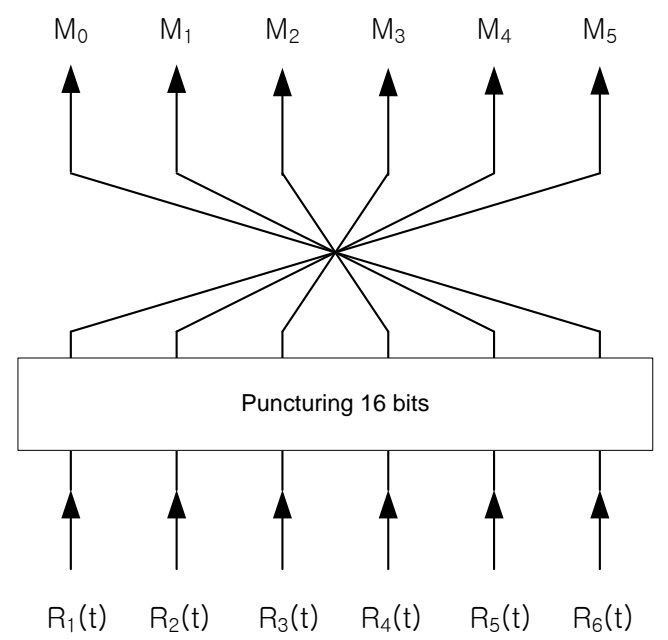

Figure 7. Generation of Basis Sequences for $(48,10)$ TFCI Code

Figure 7 shows how the basis sequences of $(48,10)$ TFCI code in TDD mode are derived from the Rademacher functions with $K=6$ of Table 2. First puncture the 16 bits in the positions of $\left\{0,4,8,13,16,20,27,31,34,38,41,44,50,54,57,61^{\text {st }}\right\}$ bits of Rademacher functions. Second the Rademacher functions $\left\{\mathrm{R}_{1}(\mathrm{t}), \mathrm{R}_{2}(\mathrm{t}), \mathrm{R}_{3}(\mathrm{t}), \mathrm{R}_{4}(\mathrm{t}), \mathrm{R}_{5}(\mathrm{t}), \mathrm{R}_{6}(\mathrm{t})\right\} \quad$ are mapped to $\left\{M_{5}(t), M_{4}(t), M_{3}(t), M_{2}(t), M_{1}(t), M_{0}(t)\right\}$, respectively. The basis sequence $M_{6}(t)$ is all "1" pattern for bi-orthogonal coding. Basis sequences $\left\{\mathrm{M}_{7}(\mathrm{t}), \mathrm{M}_{8}(\mathrm{t}), \mathrm{M}_{9}(\mathrm{t})\right\}$ are obtained by linear combination of basis sequences $\left\{M_{0}(t), M_{1}(t), M_{2}(t), M_{3}(t), M_{4}(t), M_{5}(t)\right\}$. Figure 8 is the $(48,10)$ TFCI encoder whose basis sequences are shown in Table 5. 


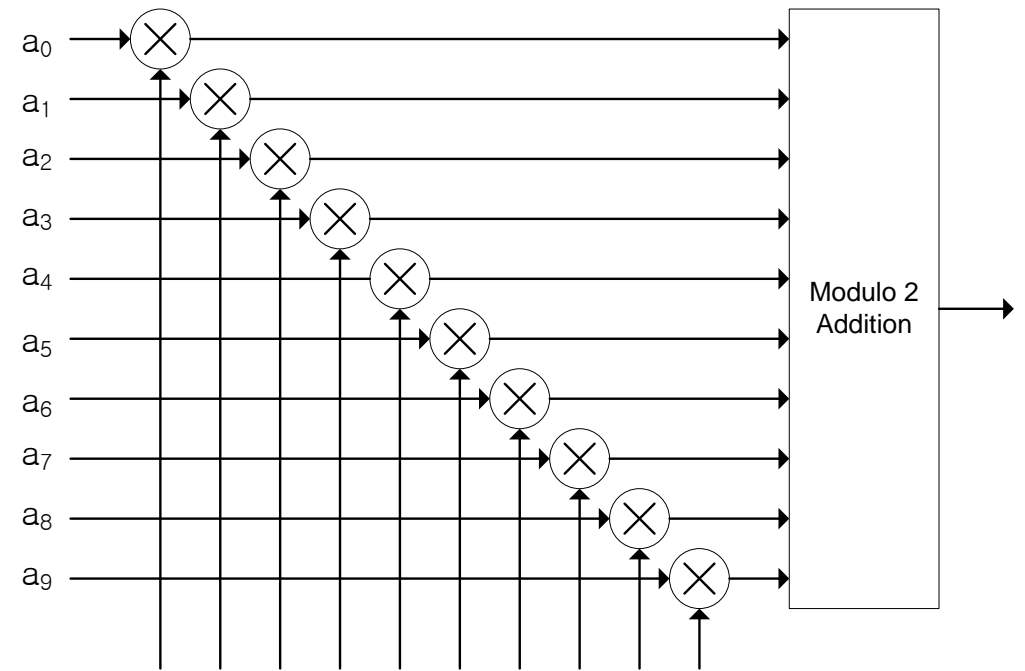

$\begin{array}{lllllllllll}M_{0} & M_{1} & M_{2} & M_{3} & M_{4} & M_{5} & M_{6} & M_{7} & M_{8} & M_{9}\end{array}$

Figure 8. $(48,10)$ TFCl Encoder 
Table 5. Basis Sequences of $(48,10)$ TFCl Code

\begin{tabular}{|c|c|c|c|c|c|c|c|c|c|}
\hline $\mathrm{M}_{0}$ & $\mathrm{M}_{1}$ & $\mathrm{M}_{2}$ & $\mathrm{M}_{3}$ & $\mathrm{M}_{4}$ & $\mathrm{M}_{5}$ & $\mathrm{M}_{6}$ & $\mathrm{M}_{7}$ & $\mathrm{M}_{8}$ & $\mathrm{M}_{9}$ \\
\hline 1 & 0 & 0 & 0 & 0 & 0 & 1 & 0 & 1 & 0 \\
0 & 1 & 0 & 0 & 0 & 0 & 1 & 1 & 0 & 0 \\
1 & 1 & 0 & 0 & 0 & 0 & 1 & 1 & 0 & 1 \\
1 & 0 & 1 & 0 & 0 & 0 & 1 & 1 & 1 & 0 \\
0 & 1 & 1 & 0 & 0 & 0 & 1 & 0 & 1 & 0 \\
1 & 1 & 1 & 0 & 0 & 0 & 1 & 1 & 1 & 0 \\
1 & 0 & 0 & 1 & 0 & 0 & 1 & 1 & 1 & 1 \\
0 & 1 & 0 & 1 & 0 & 0 & 1 & 1 & 0 & 1 \\
1 & 1 & 0 & 1 & 0 & 0 & 1 & 0 & 1 & 0 \\
0 & 0 & 1 & 1 & 0 & 0 & 1 & 1 & 0 & 0 \\
0 & 1 & 1 & 1 & 0 & 0 & 1 & 1 & 0 & 1 \\
1 & 1 & 1 & 1 & 0 & 0 & 1 & 1 & 1 & 1 \\
1 & 0 & 0 & 0 & 1 & 0 & 1 & 0 & 1 & 1 \\
0 & 1 & 0 & 0 & 1 & 0 & 1 & 1 & 1 & 0 \\
1 & 1 & 0 & 0 & 1 & 0 & 1 & 0 & 0 & 1 \\
1 & 0 & 1 & 0 & 1 & 0 & 1 & 0 & 1 & 1 \\
0 & 1 & 1 & 0 & 1 & 0 & 1 & 1 & 0 & 0 \\
1 & 1 & 1 & 0 & 1 & 0 & 1 & 1 & 1 & 0 \\
0 & 0 & 0 & 1 & 1 & 0 & 1 & 0 & 0 & 1 \\
1 & 0 & 0 & 1 & 1 & 0 & 1 & 0 & 1 & 1 \\
0 & 1 & 0 & 1 & 1 & 0 & 1 & 0 & 1 & 0 \\
0 & 0 & 1 & 1 & 1 & 0 & 1 & 0 & 1 & 0 \\
1 & 0 & 1 & 1 & 1 & 0 & 1 & 1 & 0 & 1 \\
0 & 1 & 1 & 1 & 1 & 0 & 1 & 1 & 1 & 0 \\
0 & 0 & 0 & 0 & 0 & 1 & 1 & 1 & 0 & 1 \\
1 & 0 & 0 & 0 & 0 & 1 & 1 & 1 & 1 & 0 \\
1 & 1 & 0 & 0 & 0 & 1 & 1 & 1 & 1 & 1 \\
0 & 0 & 1 & 0 & 0 & 1 & 1 & 0 & 1 & 1 \\
1 & 0 & 1 & 0 & 0 & 1 & 1 & 1 & 0 & 1 \\
1 & 1 & 1 & 0 & 0 & 1 & 1 & 0 & 1 & 1 \\
0 & 0 & 0 & 1 & 0 & 1 & 1 & 0 & 0 & 1 \\
0 & 1 & 0 & 1 & 0 & 1 & 1 & 0 & 0 & 1 \\
1 & 1 & 0 & 1 & 0 & 1 & 1 & 1 & 1 & 1 \\
1 & 0 & 1 & 1 & 0 & 1 & 1 & 0 & 0 & 1 \\
0 & 1 & 1 & 1 & 0 & 1 & 1 & 1 & 1 & 0 \\
1 & 1 & 1 & 1 & 0 & 1 & 1 & 1 & 0 & 1 \\
0 & 0 & 0 & 0 & 1 & 1 & 1 & 1 & 1 & 0 \\
1 & 0 & 0 & 0 & 1 & 1 & 1 & 0 & 1 & 1 \\
1 & 1 & 0 & 0 & 1 & 1 & 1 & 1 & 1 & 1 \\
0 & 0 & 1 & 0 & 1 & 1 & 1 & 1 & 0 & 0 \\
1 & 0 & 1 & 0 & 1 & 1 & 1 & 1 & 0 & 0 \\
1 & 1 & 1 & 0 & 1 & 1 & 1 & 1 & 1 & 1 \\
0 & 0 & 0 & 1 & 1 & 1 & 1 & 1 & 1 & 1 \\
0 & 1 & 0 & 1 & 1 & 1 & 1 & 0 & 1 & 0 \\
1 & 1 & 0 & 1 & 1 & 1 & 1 & 0 & 1 & 0 \\
0 & 0 & 1 & 1 & 1 & 1 & 1 & 0 & 1 & 1 \\
0 & 1 & 1 & 1 & 1 & 1 & 1 & 0 & 0 & 1 \\
1 & 1 & 1 & 1 & 1 & 1 & 1 & 1 & 0 & 0 \\
\hline & & & & & & & & & \\
\hline
\end{tabular}

\subsection{4. $(24,5)$ TFCI Coding}

When 2Mcps service is transmitted, 8PSK mdoulation is applied in $1.28 \mathrm{Mcps}$ TDD option [2]. If the number of TFCI bits in the range of 3 to 5, the $(24,5)$ TFCI coding is used. The basis sequences of $(24,5)$ TFCI code in TDD mode can be obtained from the Rademacher functions with $K=5$ of Table 1, whose functions 
$\left\{\mathrm{R}_{1}(\mathrm{t}), \mathrm{R}_{2}(\mathrm{t}), \mathrm{R}_{3}(\mathrm{t}), \mathrm{R}_{4}(\mathrm{t}), \mathrm{R}_{5}(\mathrm{t})\right\}$ are mapped to $\left\{\mathrm{M}_{4}(\mathrm{t}), \mathrm{M}_{3}(\mathrm{t}), \mathrm{M}_{2}(\mathrm{t}), \mathrm{M}_{1}(\mathrm{t}), \mathrm{M}_{0}(\mathrm{t})\right\}$, respectively after puncturing the first 8 bits of them. $(24,5)$ TFCI encoder is depicted in Figure 9 whose basis sequences are shown in Table 6.

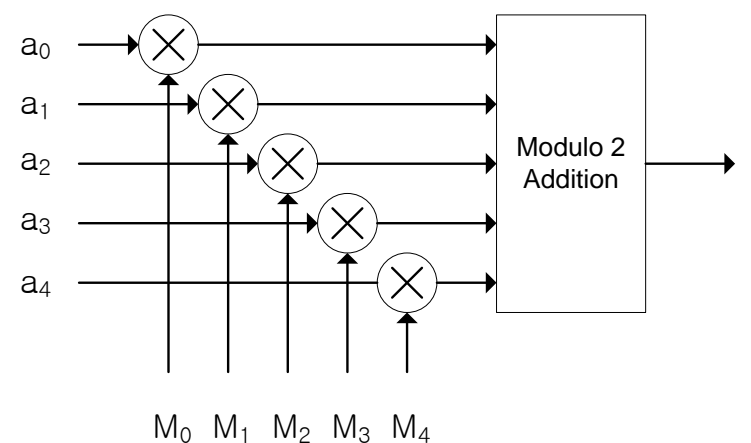

Figure 9. $(24,5)$ TFCl Encoder

Table 6. Basis sequences of $(24,5)$ TFCl code

\begin{tabular}{|c|c|c|c|c|}
\hline $\mathrm{M}_{0}$ & $\mathrm{M}_{1}$ & $\mathrm{M}_{2}$ & $\mathrm{M}_{3}$ & $\mathrm{M}_{4}$ \\
\hline 0 & 0 & 0 & 1 & 0 \\
1 & 0 & 0 & 1 & 0 \\
0 & 1 & 0 & 1 & 0 \\
1 & 1 & 0 & 1 & 0 \\
0 & 0 & 1 & 1 & 0 \\
1 & 0 & 1 & 1 & 0 \\
0 & 1 & 1 & 1 & 0 \\
1 & 1 & 1 & 1 & 0 \\
0 & 0 & 0 & 0 & 1 \\
1 & 0 & 0 & 0 & 1 \\
0 & 1 & 0 & 0 & 1 \\
1 & 1 & 0 & 0 & 1 \\
0 & 0 & 1 & 0 & 1 \\
1 & 0 & 1 & 0 & 1 \\
0 & 1 & 1 & 0 & 1 \\
1 & 1 & 1 & 0 & 1 \\
0 & 0 & 0 & 1 & 1 \\
1 & 0 & 0 & 1 & 1 \\
0 & 1 & 0 & 1 & 1 \\
1 & 1 & 0 & 1 & 1 \\
0 & 0 & 1 & 1 & 1 \\
1 & 0 & 1 & 1 & 1 \\
0 & 1 & 1 & 1 & 1 \\
1 & 1 & 1 & 1 & 1 \\
\hline
\end{tabular}

\section{CQI Coding and RTBS Coding}

In this section we discuss the relationship between CQI, RTBS, and TFCI codings.

\section{1. (20,5) CQI Coding}

The CQI is carried on the HS-DPCCH (High Speed Dedicated Physical Control Channel) to inform the base station scheduler of the data rate that the terminal is able to receive at a given time. We use Figure 10 as the encoder for $(20,5)$ CQI code whose basis 
sequences $Q_{0}, Q_{1}, Q_{2}, Q_{3}$ are exactly same as the $M_{0}, M_{1}, M_{2}, M_{3}$ of $(16,5)$ TFCI code just simply adding 4 consecutive zeros to them and $Q_{4}=M_{4}=\mathbf{1}$ [13]. The basis vector set $\left\{Q_{0}, Q_{1}, Q_{2}, Q_{3}, Q_{4}\right\}$ is as follows:

$$
\begin{aligned}
& \mathrm{Q}_{0}=[10101010101010100000] \\
& \mathrm{Q}_{1}=[01100110011001100000] \\
& \mathrm{Q}_{2}=[00011110000111100000] \\
& \mathrm{Q}_{3}=[0000000111111100000] \\
& \mathrm{Q}_{4}=[111111111111111111]
\end{aligned}
$$

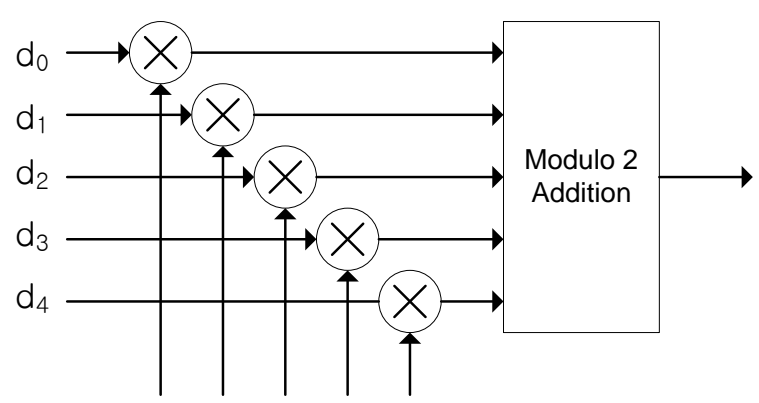

$\begin{array}{llllll}Q_{0} & Q_{1} & Q_{2} & Q_{3} & Q_{4}\end{array}$

Figure 10. $(20,5)$ CQI Encoder

\section{2. $(32,6)$ RTBS Coding}

$(32,10)$ TFCI coding is also used for encoding of RTBS (Recommended Transport Block Size) bits of CQI (Channel Quality Information) for HS-SICH (Shared Information Channel for HS-DSCH) [2]. Figure 11 is the $(32,6)$ TRBS encoder which is the simplified version of $(32,10)$ TFCI encoder with 6 basis sequences $\left\{M_{0}(t), M_{1}(t), M_{2}(t), M_{3}(t), M_{4}(t), M_{5}(t)\right\}$ of Table 3 .

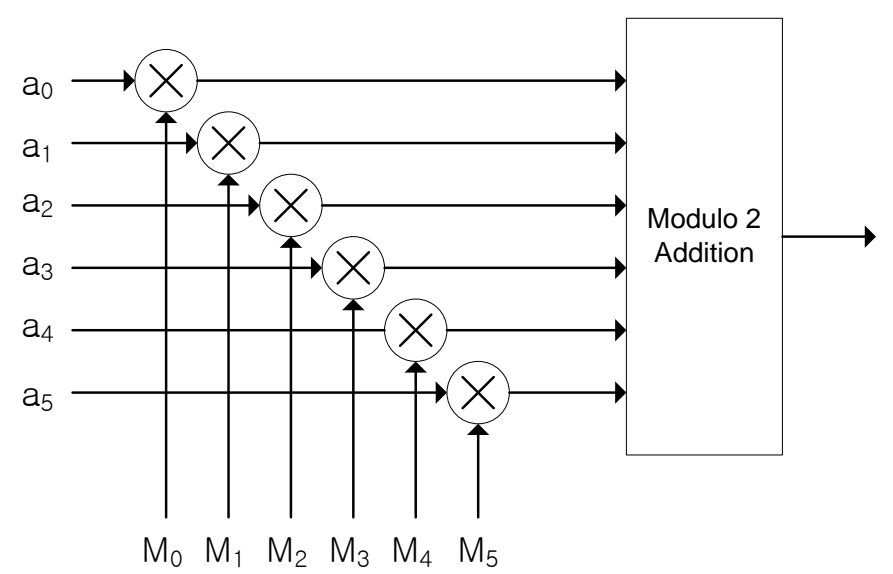

Figure 11. $(32,6)$ TFCI Encoder

\section{Conclusion}

This paper has demonstrated that the Rademacher functions are widely applied for OVSF code and $(32,10)$ TFCI, $(16,5)$ TFCI, $(24,5)$ TFCI, $(48,10)$ TFCI, $(20,5)$ CQI, $(32,6)$ RTBS codes in 3GPP FDD mode and TDD mode mobile communication systems. The OVSF codes are obtained by linearly combining the Rademacher functions. And the basis sequences of TFCI codes are constructed by using barrel shifting of Rademacher 
functions. Furthermore, we also see that $(20,5) \mathrm{CQI}$ and $(32,6)$ RTBS codings are equivalent to $(16,5)$ TFCI coding and $(32,10)$ TFCI coding, respectively.

\section{Acknowledgements}

This research was supported by the MSIP (Ministry of Science, ICT and Future Planning), Korea, under the "ICT Consilience Creative Program" (IITP-R0346-16-1007) supervised by the IITP (Institute for Information \& communications Technology Promotion.

\section{References}

[1] 3GPP TS 25.212 ver 13.0.0: Multiplexing and channel coding (FDD), Release 8, (2015).

[2] 3GPP TS 25.222 ver 13.0.0: Multiplexing and channel coding (TDD), Release 9 (2015).

[3] 3GPP TS 25.212. v4.4.0: Multiplexing and channel coding (FDD), Release 4, (2002).

[4] H. Holma and A. Toskala, "W-CDMA for UMTS", John Wiley \& Sons, Ltd, (2002).

[5] H. Holma and A. Toskala, "HSDPA/HSUPA for UMTS", John Wiley \& Sons, Ltd, (2006).

[6] F. Adachi, M. Sawahashi and K. Okawa, "Tree-structured Generation of Orthogonal Spreading Codes with Different Lengths for Forward Link of DS-CDMA Mobile Radio”, Electron. Letter, vol. 33, no. 1, (1997), pp. 27-28.

[7] F. Adachi, M. Sawahashi and H. Suda, "Wideband CDMA for Next Generation Mobile Communications Systems”, IEEE Commun. Mag., vol. 36, (1998), pp. 56-69.

[8] K. G. Beauchamp, "Walsh Functions and Their Applications", Academic Press, (1975)

[9] Y. J. Song, "Optimal Method for Encoding TFCI", Korean Patent 10-0320431, (2001).

[10] Y. J. Song, "Method for Coding and Transmitting Transport Format Combination Indicator", U.S Patent, 6,813,506, (2004).

[11] Y. J. Song, "TFCI Coding for W-CDMA System", The Journal of Korean Institute of Information Technology, vol. 7, no. 5, (2009), pp. 141-145.

[12] Y. J. Song, "Optimal Coding Scheme of Control Channels for Mobile Packet Communications", Journal of the Korea Electromagnetic Engineering Society, vol. 3, no. 1, (2003), pp. 50-56.

[13] Y. J. Song, "Basis Sequences of $(16,5)$ TFCI and (20, 5) CQI Coding in 3GPP System", Proceedings of the CES-CUBE2016, (2016).

[14] Y. J. Song, "Design of Almost Perfect Complementary Sequence Pairs", Journal of Multimedia and Ubiquitous Engineering, vol. 8, no. 6, (2014), pp. 303-312.

[15] V. K. Garg, "IS-95 CDMA and cdma2000”, Prentice Hall, Inc, (1999).

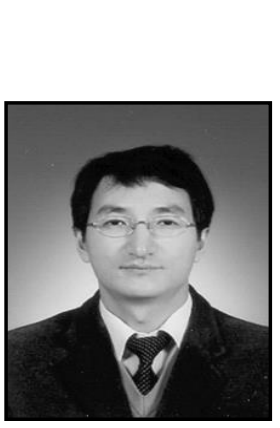

\section{Author}

Y. J. Song, he received his $\mathrm{BS}$, $\mathrm{MS}$, and $\mathrm{PhD}$ in Electronic Communications Engineering from Hanyang University, Korea, in 1987, 1994, and 1999, respectively.

$\mathrm{He}$ was a principal research engineer with LG Electronics Inc. from 1994 to 2002. Since 2002, he has been with the Department of Electronic Engineering, Kumoh National Institute of Technology, Gumi, Korea, where he is currently a Professor. During 2006, he was at the University of Hawaii at Manoa, as a visiting scholar He is the inventor of numerous international patents in 3GPP specifications regarding pilot patterns and TFCI codings. His research interests include channel coding and mobile/multimedia communication systems. 